\title{
Theory of isotopic fractionation on facetted ice crystals
}

\author{
J. Nelson \\ Laucks Foundation Inc. Suite 2100, PMB 174, 1700 Seventh Ave., Seattle, WA 98101, USA \\ Received: 8 May 2011 - Published in Atmos. Chem. Phys. Discuss.: 21 June 2011 \\ Revised: 15 October 2011 - Accepted: 30 October 2011 - Published: 16 November 2011
}

\begin{abstract}
The currently used "kinetic-fractionation" (KF) model of the differential incorporation of water-molecule isotopologues into vapor-grown ice omits surface processes on crystal facets that may be important in temperature reconstructions. This article introduces the "surface-kinetic" fractionation model, a model that includes such surface processes, and shows that differences in deposition coefficients for water isotopologues can produce isotopic fractionation coefficients that significantly differ from those of KF theory. For example, if the deposition coefficient of $\mathrm{H}_{2}^{18} \mathrm{O}$ differs by just $5 \%$ from that of ordinary water $\left(\mathrm{H}_{2}^{16} \mathrm{O}\right)$, the resulting fractionation coefficient at $20 \%$ supersaturation may deviate from the KF value by up to about $\pm 17 \%$, and even more at greater supersaturation. As a result, the surface-kinetic theory may significantly change how fractionation depends on supersaturation. Moreover, the model introduces possible new temperature dependencies from the deposition coefficients. These parameters need to be constrained by new laboratory measurements.
\end{abstract}

\section{Introduction}

Ever since the late 1950s, the fractionation of isotopes during the vapor deposition of ice has been used to make temperature reconstructions from ice cores (see e.g., Langway Jr., 2008). Reconstructions are possible because surveys in polar regions have found empirical relations between the isotopic content in surface snow and the mean surface temperature of the region. With such a relation, the measured isotopic content from ancient ice in cores extracted from the same region can be used to estimate trends in past surface temperatures (e.g., Dansgaard et al., 1969). Fundamentally, however, the

Correspondence to: J. Nelson (jontne@gmail.com) causal relation between surface temperature and surface isotopic content is complex (see e.g., Masson-Delmotte et al., 2008; Sturm et al., 2010), as it depends on the isotopic content of the oceanic vapor source, the path of the prevailing weather system, and the conditions along this path that produce fractionation to precipitating crystals. As these factors likely change during climate changes, the goal of improving temperature reconstructions from ice cores involves gaining a better understanding of the relevant processes. Here the aim is to better understand how isotopic fractionation during vapor growth to precipitating ice crystals depends on temperature and supersaturation.

The earliest fractionation theory, equilibrium fractionation, depended only on temperature. In this theory, the isotopologue sublimates from the crystal at a slower (temperature-dependent) rate than ordinary water, enriching the crystal in the heavy isotope. But in 1984, Jouzel and Merlivat, hereafter "JM", showed that this theory disagrees with the measured isotope content in surface-snow in Antarctica. By recognizing that growth was, by definition, nonequilibrium, they replaced the equilibrium fractionation coefficient with a supersaturation-dependent, nonequilibrium kineticfractionation (KF) coefficient. Then, by selecting the right cloud-supersaturation-temperature relation, their KF model could fit the surface-snow data.

But the KF coefficient ignores surface processes that are crucial to the growth of facetted crystals. And facetted crystals are common. Precipitating crystals in polar regions (e.g., Lawson et al., 2006), crystals in many cirrus and other high clouds, as well as surface hoar often consist of mainly facetted forms. Moreover, surface processes are crucial to the "surface-kinetic" model of isotopic fractionation for calcite growth from aqueous solution (DePaolo, 2011). If we include surface processes for facetted ice growth from the vapor, how much might the fractionation coefficient change? And could this change affect temperature reconstructions?

Published by Copernicus Publications on behalf of the European Geosciences Union. 
This paper develops the surface-kinetic theory of isotopic fractionation for ice growth from the vapor. Due to the influence of surface processes, the fractionation coefficient ${ }^{18} \alpha$ for isotope ${ }^{18} \mathrm{O}$ differs from the KF prediction by an amount that may be as large as $\pm 17 \%$. As described in Sect. 5, this difference could change the inferred cloud supersaturation by $20 \%$ or more, which could have a significant effect on temperature reconstructions. Thus, new experiments on ${ }^{18} \alpha$ and ${ }^{\mathrm{D}} \alpha$ (for deuterium) for facetted ice crystals are greatly needed.

\section{Background}

\subsection{Facetted growth implies regulation by surface processes}

The surface of growing atmospheric ice crystals often consists of crystalline facets, sometimes wholly so, which indicates a reduction of growth rate from surface processes (Nelson and Baker, 1996). Briefly, the vapor density adjacent to a flat surface on a small particle cannot be uniform (Frank, 1982), and the nonuniformity would produce nonuniform growth unless surface processes produce a compensating nonuniformity in the molecular rejection rate. Moreover, without such surface processes, an initially spherical frozen droplet would remain spherical as the crystal grew until being perturbed by a sufficiently large temperature or vapor-density non-uniformity, after which rounded protrusions would develop (as occurs in melt-grown ice). What we instead observe is that initially spherical frozen droplets develop facets and then grow into a great variety of facetted shapes. Thus, the existence of facets, however small, indicates the controlling influence of surface processes. And if these processes affect the incorporation of ordinary water into ice, they are likely to also affect the incorporation of water isotopologues. That is, surface processes should affect isotopic fractionation.

\subsection{Crystal growth with vapor and surface impedances}

The net vapor flux $F\left(\# \mathrm{~m}^{-2} \mathrm{~s}^{-1}\right)$ of ordinary water molecules to an ice surface is (e.g., Nelson and Baker, 1996)

$F=\frac{v}{4} \beta\left(\sigma_{\mathrm{S}}, T_{\mathrm{S}}\right)\left(N_{\mathrm{S}}-N_{\mathrm{EQ}}\right) \equiv \frac{\nu}{4} \beta\left(\sigma_{\mathrm{S}}, T_{\mathrm{S}}\right) N_{\mathrm{EQ}} \sigma_{\mathrm{S}}$,

where $v$ is the mean vapor-molecule speed, $N_{\mathrm{S}}$ is the vapor number density at the surface (molecules $\mathrm{m}^{-3}$ ), $N_{\mathrm{EQ}}$ is the equilibrium vapor number density (a function of the surface temperature $\left.T_{\mathrm{S}}\right), \sigma_{\mathrm{S}}$ is the surface supersaturation, and $\beta\left(\sigma_{\mathrm{S}}\right.$, $T_{\mathrm{S}}$ ) is the deposition coefficient function, a measure of the probability that an incident molecule to the surface reaches and attaches to a growth site on a surface step. In general, $\beta$ is nearly 0 at the lowest $\sigma_{\mathrm{S}}$ because few steps are generated, but rises to nearly 1 (assuming an efficient attachment process) as step generation increases at high $\sigma_{\mathrm{S}}$. The exact dependence on $\sigma_{\mathrm{S}}$ and $T_{\mathrm{S}}$ will depend on whether the crystal face is basal, prism, or some other orientation. Through Sect. 3, we assume all faces are identical and thus described by just one $\beta$-function. Then we consider more realistic crystals with two face types. But as the surface conditions are unknown, we must write $\sigma_{\mathrm{S}}$ and $T_{\mathrm{S}}$ in terms of the far-field (environmental) conditions $\sigma_{\infty}$ and $T$.

The surface supersaturation lies below the far-field value $\sigma_{\infty} \equiv\left(N_{\infty}-N_{\mathrm{EQ}}\right) / N_{\mathrm{EQ}}$, where $N_{\infty}$ is the far-field vapor density, by an amount that depends on how the surface and surroundings impede growth. Specifically,

$\sigma_{\mathrm{S}}=\frac{\sigma_{\infty}}{\beta\left(\sigma_{\mathrm{S}}, T_{\mathrm{S}}\right) Z}$,

where $Z$, a dimensionless number, is the total impedance to growth discussed below (Kuroda, 1984; Yokoyama and Kuroda, 1990; Nelson and Baker, 1996). Here and elsewhere, the same relations also hold for each isotopologue, whether HDO (i.e., $\mathrm{HD}^{16} \mathrm{O}$ ) or $\mathrm{H}_{2}^{18} \mathrm{O}$, except with different values of the quantities $F, v, N_{\mathrm{S}}, N_{\mathrm{EQ}}, \beta, N_{\infty}$, and $Z$. These quantities thus have superscript " $i$ ", which stands for either "D", for HDO or " 18 ", for $\mathrm{H}_{2}^{18} \mathrm{O}$. We now turn to $Z$.

The total impedance equals the sum of $Z_{\mathrm{V}}$, the vapor diffusion impedance, $Z_{\mathrm{H}}$, the thermal impedance, and $Z_{\mathrm{S}}$, the surface impedance. The vapor diffusion impedance arises from the vapor diffusing through air to the crystal surface (growth is faster without air) and increases in proportion to the crystal size times $v / D$ (see Eq. A1, Appendix A). Larger crystals are surrounded by larger vapor-depleted regions and thus have greater impedance. As an example, at sea-level pressure, a spherical crystal starting at $1-\mu \mathrm{m}$ radius and ending at $500 \mu \mathrm{m}$ would have a $Z_{\mathrm{V}}$ value increasing from 7.5 to 3700. At lower pressures, $Z_{\mathrm{V}}$ decreases in proportion to the pressure decrease. The thermal impedance arises from the temperature rise of the crystal, the temperature at which the latent heating balances thermal diffusion to the surrounding air. Its magnitude decreases rapidly with decreasing temperature (in proportion to $N_{\mathrm{EQ}}$ ) and is less than $Z_{\mathrm{V}}$ below about $-5^{\circ} \mathrm{C}$ (Nelson and Baker, 1996). So to simplify the expressions, we drop $Z_{\mathrm{H}}$ (though it can easily be added to $Z_{\mathrm{V}}$ ) and assume $T_{\mathrm{S}}=T$.

The surface impedance equals the inverse of the deposition coefficient:

$Z_{\mathrm{S}}\left(\sigma_{\mathrm{S}}, T\right) \equiv \frac{1}{\beta\left(\sigma_{\mathrm{S}}, T\right)}$

(for brevity, we often drop the dependence on $\sigma_{\mathrm{S}}$ and $T$ ). This impedance results from an increase in surface-mobile molecules that desorb from the surface. The number of such molecules per area of surface exceeds the equilibrium value because the supersaturated vapor produces a greater-thanequilibrium flux of molecules to the surface and some of the excess molecules do not reach (and incorporate into) a strong-binding site on a surface step. That is, a molecule may fail to reach a step, or having reached a step, fail to 
bind to the step. For surface processes to control growth, the fraction of the incident molecules that reach a step should be significantly below unity, meaning that the average step spacing likely far exceeds the distance such a water molecule migrates before desorbing.

\section{Basic theory of surface-kinetic fractionation}

Under constant conditions, the ratio $\chi$ of the number of isotopologue molecules to $\mathrm{H}_{2} \mathrm{O}$ molecules in the crystal equals the ratio of their respective net vapor fluxes to the surface. From Eqs. (1) and (2), this ratio equals

${ }^{i} \chi=\frac{\left({ }^{i} N_{\infty}-{ }^{i} N_{\mathrm{EQ}}\right)}{N_{\mathrm{EQ}} \sigma_{\infty}{ }^{i} d} \frac{1+z}{1+{ }^{i} z}$,

where $z \equiv Z_{\mathrm{S}} / Z_{\mathrm{V}}$ and ${ }^{i} d \equiv D /{ }^{i} D$, the ratio of the vapor diffusion constants. (In general, $\chi$ will vary during growth as conditions change.) But, by definition of the equilibrium fractionation coefficient ${ }^{i} \alpha_{\mathrm{S}}$ (JM, Eq. 7), the corresponding isotopic number ratio in the vapor differs from that in the solid by the equilibrium fractionation ratio for ice:

$\frac{{ }^{i} N_{\mathrm{EQ}}}{N_{\mathrm{EQ}}}=\frac{{ }^{i} \chi}{{ }^{i} \alpha_{\mathrm{S}}}$.

(Formulas in Jouzel (1986) for ${ }^{i} \alpha_{\mathrm{S}}$ are $\ln ^{18} \alpha_{\mathrm{S}}=$ $11.839 / T-28.224 \times 10^{-3}$ and $\ln ^{\mathrm{D}} \alpha_{\mathrm{S}}=16288 / T^{2}-9.34 \times$ $10^{-2}$, which are from the original experiments of Majoube (1970) and Merlivat and Nief, 1967). We define the nonequilibrium fractionation coefficient ${ }^{i} \alpha$ like that in Eq. (5) except with the far-field, non-equilibrium, vapor density:

$\frac{{ }^{i} N_{\infty}}{N_{\infty}}=\frac{{ }^{i} \chi}{{ }^{i} \alpha}$.

Using Eqs. (4), (5), and (6) to eliminate ${ }^{i} N_{\mathrm{EQ}},{ }^{i} N_{\infty}$, and ${ }^{i} \chi$, one gets

${ }^{i} \alpha=\frac{1+\sigma_{\infty}}{\frac{1}{i_{\alpha S}}+\sigma_{\infty}{ }^{i} d \frac{1+{ }^{i} z}{1+z}}$,

which is our fundamental result. Hereafter, to reduce the amount of notation, the superscript " $i$ " will be removed unless needed (e.g., to distinguish $z$ from ${ }^{i} z$ and $\beta$ from ${ }^{i} \beta$ ) or if a result pertains only to one isotopologue.

Three limits of Eq. (7) stand out: the equilibrium limit, the KF limit, and the surface-kinetic limit. (Regimes 1-3 in DePaolo's (2011) terminology.) In the first, $\alpha \rightarrow \alpha_{\mathrm{S}}$ when $\sigma_{\infty} \rightarrow 0$. In the KF limit, the surface impedances vanish $\left(z,{ }^{i} z \rightarrow 0\right)$ giving

$\alpha_{\mathrm{KF}}=\frac{1+\sigma_{\infty}}{\frac{1}{\alpha_{\mathrm{S}}}+\sigma_{\infty} d}$,

which shows that KF fractionation occurs whenever $d \neq$ $1 / \alpha_{S}$. Equation (8) agrees with JM's result, though they wrote the equivalent expression as $\alpha_{\mathrm{K}} \cdot \alpha_{\mathrm{S}}$. (Fisher (1991) does a more detailed analysis of the temperature difference between crystal and air, but the result is nearly indistinguishable from the KF result.) Finally, in the surface-kinetic limit $\left(z,{ }^{i} z \gg 1\right)$, writing $x \equiv \beta /{ }^{i} \beta$ and $y \equiv v /{ }^{i} v$

$\alpha_{\mathrm{SK}}=\frac{1+\sigma_{\infty}}{\frac{1}{\alpha_{\mathrm{S}}}+\sigma_{\infty} y x}$,

showing that surface fractionation occurs when $x \neq 1 / y \alpha_{S}$ and the results should deviate from the KF case when $x \neq$ $d / y$. Values of these quantities are in Table 1.

Thus, fractionation depends on four factors: $\alpha_{\mathrm{S}}, d, y$, and $x$. Physically, $\alpha_{\mathrm{S}}$ arises from different isotopic rates of desorption of an equilibrium distribution of water species on the ice surface. But under supersaturated conditions, vapor flows to the ice surface, producing additional fractionation due to different isotopic rates of vapor diffusion $(d)$, molecular impingement to the surface $(y)$, and desorption from the surface $(x)$. The isotopic desorption rates change because the surface has a greater-than-equilibrium concentration of mobile water species; the species with a lower deposition coefficient will have a greater increase in mobile molecules on the surface, and thus a corresponding increase in desorption rate. Finally, of these factors, the first three are nearly unity (Table 1) and independent of supersaturation. However, $x$ may vary with supersaturation and temperature, but is presently unknown. Here, we assume a range of $0.8 \leq x \leq 1.2$ as described in Appendix B.

For surface-kinetic fractionation to be significant, the surface impedance must roughly equal or exceed the vapor impedance. To determine the surface impedance, we must estimate $\beta$, which depends on $\sigma_{\mathrm{S}}$ and $T$. We can approximate various functional forms using two parameters $\sigma_{1}$ and $n$ as

$\beta\left(\sigma_{\mathrm{S}}, T\right)=\left(\frac{\sigma_{\mathrm{S}}}{\sigma_{1}(T)}\right)^{n(T)}$,

where $n>0$ and $\sigma_{1}$ is a characteristic supersaturation that depends on temperature and surface properties of the crystal facet. (We do not use an analogous equation for ${ }^{i} \beta$ because Eq. (10) reflects the generation of growth steps, which is controlled by ordinary water, the dominant molecular species. For ${ }^{i} \beta$, we assume a fixed $x$ as discussed in Appendix B.) Equation (10) is a simplification of that introduced in Nelson and Baker (1996), but shows the same basic features; $n=1$ describes growth via a single screw-dislocation when the airpressure is low, and large $n$ describes growth by layer nucleation. For example, in a study of critical supersaturations for layer-nucleation growth, $\sigma_{1}$ varied between 0.0015 and 0.025 for the basal face as the temperature decreased between -1 and $-16^{\circ} \mathrm{C}$ (Nelson and Knight, 1998). The value may be higher at lower temperatures. (Nelson and Baker (1996) included a second factor in Eq. (10), but its omission has negligible effect in atmospherically relevant calculations.) For a 
Table 1. Fractionation factors in Eqs. (8) and (9).

\begin{tabular}{lllllll}
\hline & $\alpha_{\mathrm{S}}^{-1}\left(0^{\circ} \mathrm{C}\right)$ & $\alpha_{\mathrm{S}}^{-1}\left(-20^{\circ} \mathrm{C}\right)$ & $y^{*}$ & $d^{* *}$ & $\left(y \alpha_{\mathrm{S}}\right)^{-1}\left(-20^{\circ} \mathrm{C}\right)$ & $d / y$ \\
\hline $\mathrm{H}_{2}^{18} \mathrm{O}$ & 0.985 & 0.982 & 1.054 & 1.029 & 0.932 & 0.976 \\
\hline $\mathrm{HDO}$ & 0.883 & 0.852 & 1.028 & 1.025 & 0.829 & 0.997 \\
\hline
\end{tabular}

* Calculated using $v \propto 1 / \sqrt{ }$ mass.

** From Merlivat (1978). Newer values are 1.032 and 1.016 (Cappa et al., 2003).

specific set of conditions, the values of $\beta$ and $\sigma_{\mathrm{S}}$ are determined by combining Eq. (10) with Eqs. (2) and (3). When $n$ equals 1 or 2 , one can solve for $\beta$ analytically and deduce $\sigma_{\mathrm{S}}$, but cases with larger $n$ (e.g., layer-nucleated steps) requires a numerical method. Numerical results are described in Appendix $\mathrm{C}$ and used in the calculation of $z$ for Fig. 1. We now show the results.

Of the two variable factors that affect $\alpha$, the surface impedance ratio $z$ can vary the most. As shown in Fig. 1, $z$ decreases with increasing crystal size (because $Z_{\mathrm{V}}$ increases), and by comparing curves, note that $z$ also decreases when $\sigma_{\infty}$ increases (with $\sigma_{1}$ fixed). Conversely, $z$ increases with an increase in either $\sigma_{1}$ or $n$ (because $Z_{\mathrm{S}}$ increases). $z$ also increases with elevation due to the air-pressure dependence of the diffusion constant.

Large $z$ values produce $\alpha$ values that deviate from the $\mathrm{KF}$ prediction. For example, at relatively high surface impedance, as in the upper "beaded" curve in Fig. $2(z>3$ over the entire supersaturation range), the fractionation lies above the KF value because $x=0.95$, which is less than $d=1.03$. But, as $x$ is not below $1 / \alpha_{\mathrm{S}}$, the fractionation does not exceed the equilibrium value, instead lying roughly halfway between the KF and equilibrium values. In general, when $x<1$, the deposition coefficient of the isotopologue exceeds that of regular water, making the ice richer in isotope by an amount that depends on $z$. Similarly, when $x>d$, the surface fractionation acts in the same direction as KF, driving the degree of fractionation even lower. For example, when $x$ is instead 1.05 , the fractionation lies distinctly below the KF curve (Fig. 2, lower beaded curve). In this case, the fractionation from $y$ acts together with that from $x$, increasing the effect.

If $x$ deviates further from unity, $z$ need not be large for surface fractionation to have a large effect. For example, with middling values of $z$, the fractionation exceeds $\alpha_{S}$ when $x=0.8$ (Fig. 2, top curve). And when $x=1.2$, the surface fractionation may lie below the KF value by an amount nearly double the amount KF lies below the equilibrium value (Fig. 2, bottom curve). In contrast, at low surface impedance, the fractionation remains close to the KF value even when the $x$ value deviates $20 \%$ from unity.

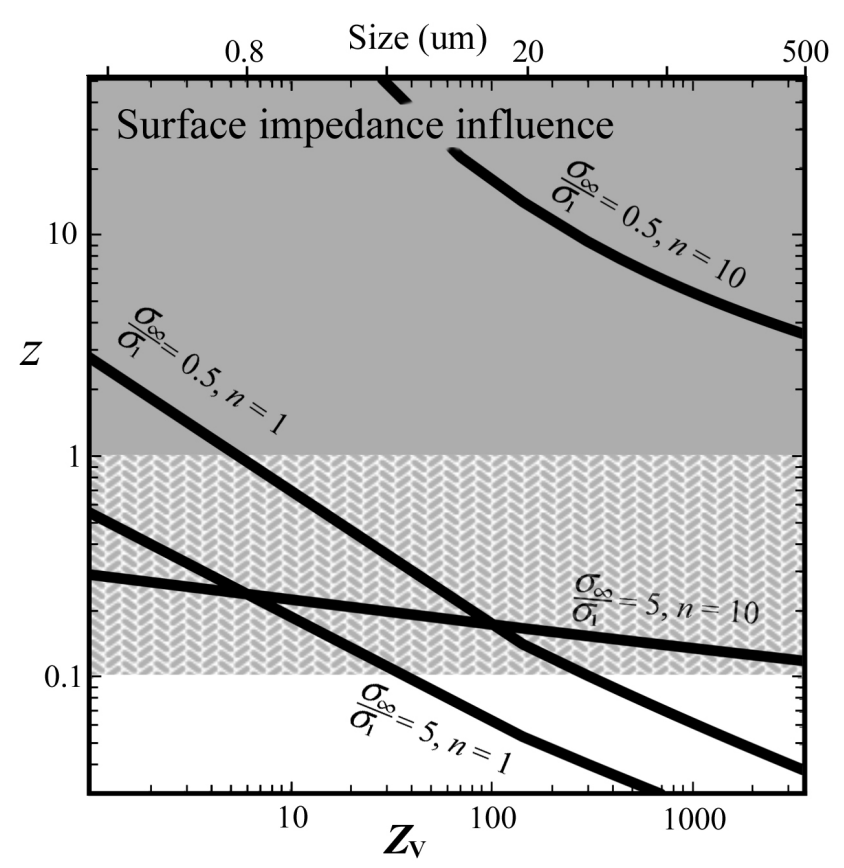

Fig. 1. Relative influence of the surface impedance for a range of vapor impedances (from Eqs. C1, C2). Each curve represents a crystal with the labeled surface parameters. Crystals in the grey zone $(z \geq 1)$ likely have fractionation values significantly affected by surface processes, whereas those in the white zone $(z \leq 0.1)$ will likely not be affected. The case for the hatched region depends on the ratio $x$. Crystal diameter values on the top scale assume the equation for $Z_{V}$ of a sphere at 1000 mbar (see Appendix A).

\section{Surface-kinetic fractionation to realistic crystals}

\subsection{Cylindrical crystals}

We now make the model more realistic by considering crystals shaped as tabular or columnar cylinders. In addition to introducing the variable height/width ratio (aspect ratio), the cylinder case has two distinct faces, with the top/bottom, or "basal" face having fractionation value $\alpha_{\mathrm{B}}$, and the side or "prism" face having value $\alpha_{\mathrm{P}}$.

The formulas for $\alpha_{\mathrm{B}}$ and $\alpha_{\mathrm{P}}$ follow from Eq. (7) with the appropriate substitution; for example, for $\alpha_{\mathrm{B}}$, we substitute $z_{\mathrm{B}}$ for $z$ and ${ }^{i} z_{\mathrm{B}}$ for ${ }^{i} z$. Concerning $z_{\mathrm{B}}$ and $z_{\mathrm{P}}$, the surface 


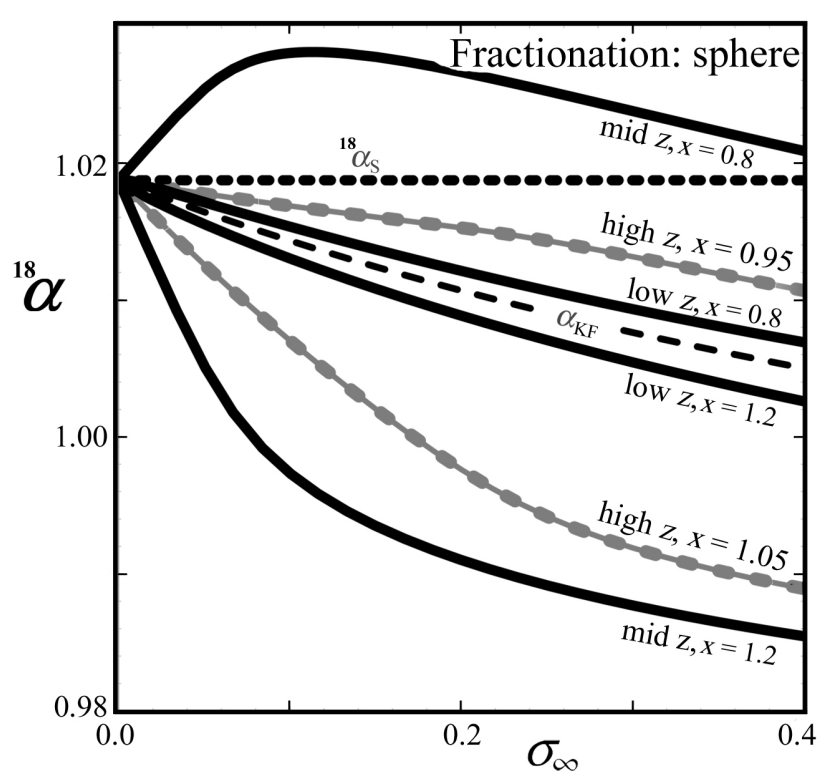

Fig. 2. Calculated $\mathrm{H}_{2}^{18} \mathrm{O}$ fractionation coefficients ${ }^{18} \alpha$ for completely facetted ice growing from the vapor at $-20^{\circ} \mathrm{C}$, a fixed crystal size, and a range of supersaturations $\sigma_{\infty}$. Dotted and dashed lines show equilibrium and KF fractionation values. Deviations from the KF prediction depend on $x=\beta /^{i} \beta$, which is assumed constant, and $z=Z_{\mathrm{S}} / Z_{\mathrm{V}}$, which decreases with increasing $\sigma_{\infty}$. Light, beaded curves have the highest $z$ values considered here, exceeding 3.1 at all $\sigma_{\infty}\left(Z_{\mathrm{V}}=100, \sigma_{1}=0.5, n=10\right)$. The two "low $z$ " curves have $z$ values below 0.1 when $\sigma_{\infty} \geq 0.05\left(Z_{\mathrm{V}}=1000\right.$, $\left.\sigma_{1}=0.2, n=1\right)$. The uppermost and lowermost curves have middling $z$ values, exceeding 1 when $\sigma_{\infty} \leq 0.2\left(Z_{\mathrm{V}}=1000, \sigma_{1}=0.4\right.$, $n=5$ ).

impedances equal the reciprocals of $\beta_{\mathrm{B}}$ and $\beta_{\mathrm{P}}$, just as in the sphere case, but the vapor impedances are more complex, depending not only on crystal size, but also on shape and rate of shape change as discussed in Appendix A.

To determine values for $\alpha_{\mathrm{B}, \mathrm{P}}$, one must know the deposition coefficients, which means determining $\sigma_{\mathrm{S}}$. But with a nonspherical crystal such as the cylinder, $\sigma_{\mathrm{S}}$ varies along the surface, so which $\sigma_{\mathrm{S}}$ value determines $\beta$ ? The appropriate $\sigma_{\mathrm{S}}$ value for $\beta$ (in Eq. 10) is the point of highest $\sigma_{\mathrm{S}}$ because this point determines the growth rate (e.g., Wood et al., 2001). This point is usually the edge of the facet (unless some face has essentially stopped growing, Nelson, 2001). Here, we assume this is the case for both the basal and prism facets. As a result, the $\sigma_{\mathrm{S}}$ value solves

$\sigma_{\mathrm{S}}=\frac{\sigma_{\infty}}{1+\beta_{\mathrm{B}}\left(\sigma_{\mathrm{S}}, T\right) Z_{\mathrm{VB}}}=\frac{\sigma_{\infty}}{1+\beta_{\mathrm{P}}\left(\sigma_{\mathrm{S}}, T\right) Z_{\mathrm{VP}}}$,

which is similar to Eq. (2).

To get the mass-averaged $\alpha$, one multiplies each coefficient by the mass-uptake (flux times facet area) on the corresponding facets:
$\alpha=\alpha_{\mathrm{B}} \frac{\gamma}{\gamma+2 \Gamma}+\alpha_{\mathrm{P}} \frac{2 \Gamma}{\gamma+2 \Gamma}$,

where $\gamma \equiv \beta_{\mathrm{B}} / \beta_{\mathrm{P}}$ is the growth-rate ratio (Nelson and Baker, 1996). For example, in steady-state, $\gamma=\Gamma$, and thus $2 / 3$ of the mass enters via the prism faces. But in general, a range of fractionation values can occur, depending on the crystal aspect ratio, the growth-rate ratio, and the fractionation to each face. The last factor depends on the ratios of the deposition coefficient functions $x_{\mathrm{B}} \equiv \beta_{\mathrm{B}}{ }^{i} \beta_{\mathrm{B}}$ and $x_{\mathrm{P}} \equiv \beta_{\mathrm{P}} /{ }^{i} \beta_{\mathrm{P}}$.

The results show that the crystal shape affects fractionation at high $z$, but mostly through the parameters $x_{\mathrm{B}}$ and $x_{\mathrm{P}}$. For example, when $z>2.5$ (all solid curves in Fig. 3), yet both facets have the same $x$ ratio of 1.05 , the fractionation coefficient is only slightly less than the sphere result - the sphere and cylinder results are nearly identical. This is shown by curve 1 in which $\Gamma=\gamma=10$. At larger $\Gamma$, the fractionation coefficient decreases further, though the effect remains relatively small. Larger influences on $\alpha$ can occur when $x_{\mathrm{B}} \neq x_{\mathrm{P}}$. In particular, for steady-state growth $(\gamma=\Gamma)$ with $\Gamma=10$, fractionation decreases when $x_{\mathrm{P}}>x_{\mathrm{B}}$ (curve 2), even though their average still equals 1.05 because in steadystate, most mass enters through the prism face, which has an $x_{\mathrm{P}}$ value of 1.1 .

However, growth is rarely steady. Instead, $\Gamma$ deviates further and further from unity during growth (Takahashi et al., 1991), meaning $\gamma \geq \Gamma$ for columns and $\gamma \leq \Gamma$ for plates. In the non-steady-state case of curve 3 , most mass enters through the basal face, which has $x_{\mathrm{B}}=1.1$, bringing the curve lower. Similarly, when most of the mass enters through the prism face and $x_{\mathrm{P}}=1.1$, as in the tabular-crystal case in curve 4 , then the fractionation coefficient is significantly below that of the sphere. These cases (2-4) show deviations in $\alpha$ below that of an equivalent sphere of $x=1.05$ because the face with most of the mass uptake had $x>1.05$. If instead they had $x<1.05$, then the resulting $\alpha$ value would be above that of a sphere.

These cylinder results emphasize what we found with the sphere: when the surface impedance dominates, small changes in $x$ can introduce relatively large variations in fractionation coefficient. For the cylinder, this applies to small changes in $x$ on the facets that dominate growth. As the crystal shape itself has little influence on fractionation, this result should apply to polycrystals and any other crystal bound by facets.

\subsection{Incompletely facetted crystals}

Stellar and hollowed crystals are incompletely facetted, meaning that some of the mass uptake comes from nonfacetted (NF) regions. For this case, we need an extra term in Eq. (12):

$\alpha=\alpha_{\mathrm{B}} M_{\mathrm{B}}+\alpha_{\mathrm{P}} M_{\mathrm{P}}+\alpha_{\mathrm{NF}} M_{\mathrm{NF}}$, 


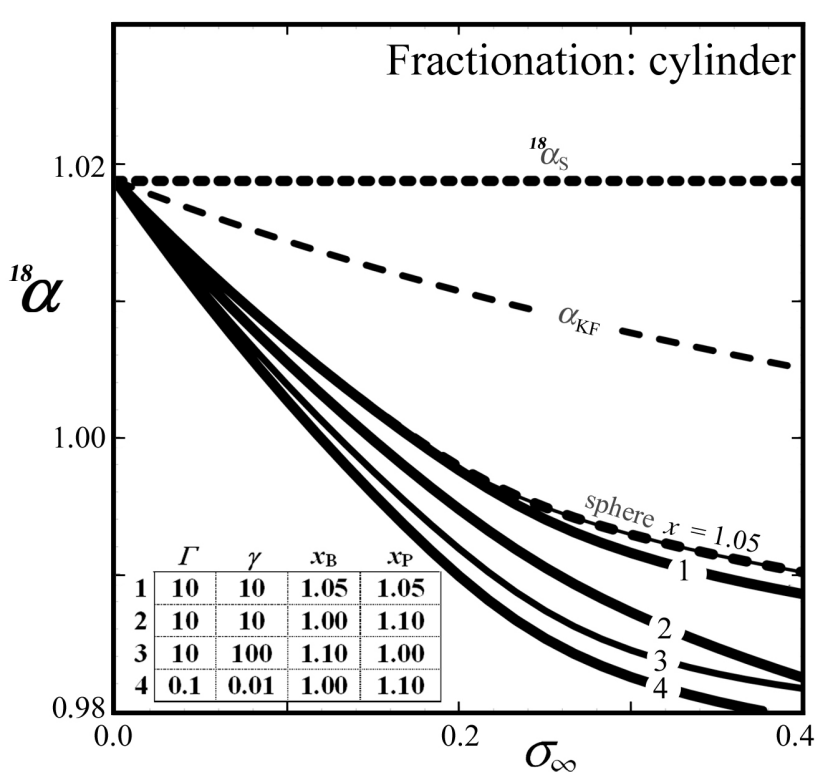

Fig. 3. Fractionation coefficient ${ }^{18} \alpha$ for fully facetted cylindrical crystals at $T=-20^{\circ} \mathrm{C}$. All cases had the same average $x$, the same volume (giving $Z_{\mathrm{V}}=300$ for the equivalent sphere) and growth parameters $\sigma_{1}=0.5$ and $n=10$ on the fastest-growing face (same as beaded curves in Fig. 2). Cases 1-3 are columnar crystals with $\Gamma$ $=10$, whereas case 4 is a tabular crystal with $\Gamma=0.1$. Crystals in cases $1 \& 2$ grow under steady-state growth, whereas $3 \& 4$ grow with increasing shape anisotropy. For cases $2-4$, switching values of $x_{\mathrm{B}}$ and $x_{\mathrm{P}}$ would yield curves above that shown for the sphere.

where $M_{j}$ stands for the fraction of mass uptake that occurs through face type " $j$ " and $\alpha_{\mathrm{NF}}$ equals the fractionation coefficient for non-facetted regions. The latter coefficient should equal $\alpha$ in the limit $\beta \rightarrow 1$, and thus nearly equal $\alpha_{\mathrm{KF}}$.

For stellar or dendritic crystals, it is hard to accurately estimate $M_{\mathrm{P}}$ and $M_{\mathrm{NF}}$ without newer, more careful measurements. I attempted such an estimate in Nelson (2005), using the measurements of Takahashi et al. (1991), and found that $M_{\mathrm{NF}}$ varied between 0.77 and 0.87 for crystals between -13.3 and $-16.8^{\circ} \mathrm{C}$. Thus, most of the mass uptake on such crystals occurs on the non-facet regions.

A similar difficulty occurs with hollowed columns, except the problem instead lies in estimating $M_{\mathrm{B}}$ and $M_{\mathrm{NF}}$. However, if we assume that the hollowed regions are cylindrical cones extending to the crystal center, and if the volume of the hollows remain a fixed fraction $K$ of the volume of the equivalent non-hollowed crystal, then the resulting mass-uptake fractions can be shown to equal

$$
\begin{aligned}
& M_{\mathrm{B}}=\frac{\gamma}{\gamma+2 \Gamma} \frac{1-3 K}{1-K}, \\
& M_{\mathrm{P}}=\frac{2 \Gamma}{\gamma+2 \Gamma} \frac{1}{1-K}, \\
& M_{\mathrm{NF}}=\frac{2 K}{\gamma+2 \Gamma} \frac{\gamma-\Gamma}{1-K} .
\end{aligned}
$$

When the hollow extends across the entire basal face, $K$ has its maximum value of $1 / 3$. In this case, $M_{\mathrm{B}}=0$ and the fraction of mass uptake by the non-facet region $M_{\mathrm{NF}}$ has its maximum value, a value that depends on $\gamma / \Gamma$. Using the measurements for hollow columns at $-5.3^{\circ} \mathrm{C}$ from Takahashi et al., 1991, $\gamma / \Gamma=5.4$, giving $M_{\mathrm{NF}}=0.6$. As the hollows did not appear to extend across the basal faces, this value may be an overestimate. Nevertheless, significant amounts of uptake likely occur in the non-facet regions of hollow columns.

\section{Discussion}

\subsection{Is surface-kinetic fractionation consistent with Antarctic snow composition?}

We typically measure the isotopic content by $\delta$, the relative deviation of the fractional amount of isotope in ice from the SMOW (standard mean ocean water) standard (i.e., $\delta X$ $\left.\equiv\left(X_{\mathrm{r}}-X_{\mathrm{r}-\mathrm{SMOW}}\right) / X_{\mathrm{r}-\mathrm{SMOW}}\right)$, where $X_{\mathrm{r}}$ is the ratio of isotopologue to ordinary water). Many measurements of $\delta^{18} \mathrm{O}$ have been made at the snow surface in Antarctica over regions spanning a wide range of average annual temperature. According to the recent review of Masson-Delmotte et al. (2008), coastal regions, with a mean ground-surface temperature $T_{\mathrm{g}}$ of about $-10^{\circ} \mathrm{C}$, have a mean $\delta^{18} \mathrm{O}$ level of about $-15 \%$, whereas inland plateau regions, with a mean temperature near $-60{ }^{\circ} \mathrm{C}$, have $\delta^{18} \mathrm{O}$ near $-55 \%$. The bestfit relation is

$\delta^{18} \mathrm{O}[\% \mathrm{o}]=0.8 T_{\mathrm{g}}-8.11$,

with individual data points varying from the relation by about $\pm 7 \%$. (Other regions have a different relation. See e.g., Johnsen et al., 1989.) We now ask if the new surface-kinetic $\alpha$ is consistent with the above relation.

Here we follow the procedure in JM and show that $\alpha$ can be made consistent with Eq. (15) by finding a reasonable supersaturation-temperature curve. As in JM, assume a Rayleigh process in which ice crystals grow solely by vapor deposition. Specifically, as a parcel of air with vapor mixing ratio $m_{\mathrm{v}}$ and temperature $T$ cools and precipitates, the isotopic content of the new precipitate changes as (see e.g., JM, Salamantin et al., 2004)

$$
\frac{d \delta}{d T} \approx(1+\delta)(\alpha-1) \frac{d \ln \left[m_{\mathrm{v}}\right]}{d T},
$$

where a much smaller term $(\alpha d \alpha / d T)$ has been dropped. To determine a $\delta^{18} \mathrm{O}-T$ relation, one must integrate Eq. (16) along the condensation path from an initial temperature to a colder, final temperature, specifying the temperaturedependence of the supersaturation and air pressure. As in $\mathrm{JM}$, we will assume the air parcel travels along a temperature inversion, beginning at $-10^{\circ} \mathrm{C}$, gaining elevation and cooling as it moves inland such that $P[\mathrm{mb}]=1095+19.14 T+$ $0.1857 T^{2}$. At the inversion, the temperature relates to the 
ground temperature as $T\left[{ }^{\circ} \mathrm{C}\right]=0.67 T_{\mathrm{g}}-1.2$. With this relation, one can convert the integrated $\delta^{18} \mathrm{O}-T$ curve to a $\delta^{18} \mathrm{O}$ $T_{\mathrm{g}}$ curve to compare with Eq. (15). By following this procedure, and adjusting the supersaturation-temperature relation, I fit the data in Eq. (15).

The general trend in the resulting supersaturation can be readily estimated. For the above trajectory, the rightmost factor in Eq. (16) ranges from approximately $0.07^{\circ} \mathrm{C}^{-1}$ at $-10^{\circ} \mathrm{C}$ to $0.12^{\circ} \mathrm{C}^{-1}$ at $-50^{\circ} \mathrm{C}$, being nearly independent of supersaturation. Given that $1+\delta \approx 1$, this means that, to give the slope of $0.8 \% 0^{\circ} \mathrm{C}^{-1}$ in Eq. (15), $\alpha-1$ must decrease from about 0.017 to about 0.010 over the same temperature change. This decrease in $\alpha$ means that the supersaturation should increase, but at a rate that depends on the slope of $\alpha\left(\sigma_{\infty}\right)$.

For the three cases considered, the fitted supersaturation curves lie below the liquid-water saturation value for all but the lowest temperatures (Fig. 4). Such supersaturations represent averages over the growth of a crystal, and thus would occur when the crystals are initially surrounded by many droplets that later evaporate. Conversely, the supersaturation may be steady, yet contain little-to-no liquid water. With the KF kinetic-fractionation coefficient, the supersaturation curve in the figure is roughly one-half of the liquid-water value, which is consistent with the analysis in JM. But with the surface-kinetic fractionation curve for $x=0.95$, the $\alpha$ value at a given $\sigma_{\infty}$ is larger (see Fig. 2), which means that to have the same $\alpha$ value, the supersaturation value must be larger. Thus the curve for this case is higher. For the same reason, the supersaturation curve for $x=1.05$ lies below that of the KF case.

These surface-kinetic examples $\alpha\left(\sigma_{\infty}\right)$ represent idealizations; in general, the value of $\alpha$ will also vary depending on the degree of faceting, the crystal size, and any explicit temperature or supersaturation dependences of the surface parameters. Still, the examples help constrain the likely range of $x$. For example, of the two cases, $x=1.05$ appears more consistent with observations than $x=0.95$ for two reasons: (i) the large value of $z$, which was assumed, is unlikely at the high supersaturation needed in the $x=0.95$ case (Fig. 1 shows $z$ to decrease when $\sigma_{\infty}$ increases), and (ii) the cloud supersaturation cannot exceed liquid-water saturation, as it does for the $x=0.95$ case (and below the homogeneous freezing point at $-40^{\circ} \mathrm{C}$, it must lie below liquid water saturation). Moreover, at Dome Fuji, where the mean annual temperature is about $-55^{\circ} \mathrm{C}$, about $53 \%$ of the precipitation is from diamond dust (Fujita and Abe, 2006), which likely grew at relatively low supersaturations. And the low supersaturations are consistent with the $x=1.05$ case. Finally, the average supersaturation slope of the $x=1.05$ case equals $0.0028^{\circ} \mathrm{C}^{-1}$, which is close to the slopes of 0.0038 and $0.0020^{\circ} \mathrm{C}^{-1}$ that Masson-Delmotte et al. (2008) used to fit surface data. Therefore, surface-kinetic fractionation is consistent with measured $\delta^{18} \mathrm{O}$ trends, and the analysis suggests that $x \geq 1$.

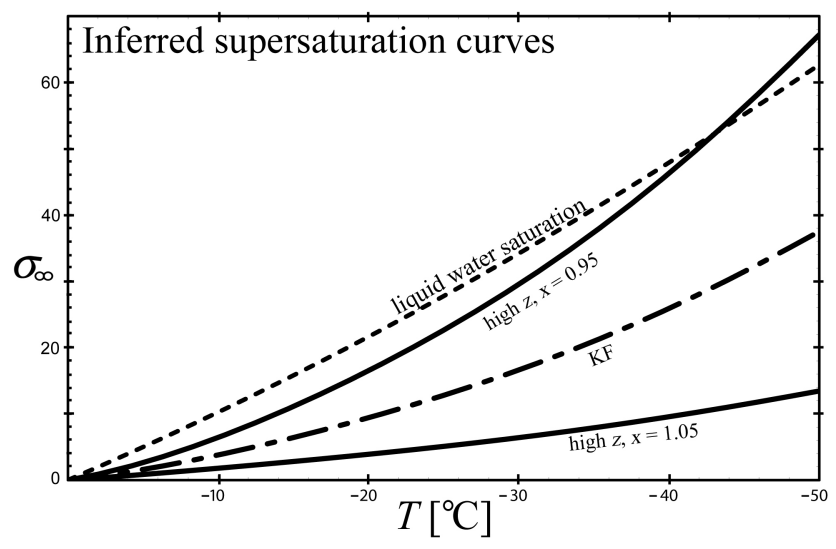

Fig. 4. Inferred supersaturation dependence to fit surface measurements of $\delta^{18} \mathrm{O}$ (Eq. 15). The three lower curves, when used in the integration of Eq. (16) with the $\alpha$ functions of Fig. 2 of the same label, result in $\delta^{18} \mathrm{O}$ values equal to those in Eq. (15). The top curve is the supersaturation of a cloud of water droplets.

The surface-kinetic aspects of fractionation can add, or subtract, to the sensitivity that $\delta$-values in precipitation have to supersaturation. To address how a variation in average supersaturation could, through the change in $\alpha$ during fractionation, produce scatter from the curve given by Eq. (15), notice that the predicted change will equal the change in temperature $\Delta T_{\mathrm{g}}$ times the change in slope $(\Delta d \delta / d T)$ caused by the supersaturation change (neglecting other factors that may change $\alpha$ ). Using Eq. (16) and setting $1+\delta$ to 1 , this is

$\Delta \delta[\% o] \approx 1000 \cdot 0.67 \Delta T_{\mathrm{g}} \Delta \sigma \frac{d \alpha}{d \sigma} \frac{d \ln \left[m_{\mathrm{v}}\right]}{d T}$,

where the factor of 1000 arises from the conversion to \%o units and the 0.67 from the conversion to ground temperature. The next factors $\Delta T_{\mathrm{g}} \Delta \sigma$ represent the average variation in supersaturation for precipitation that fell to the ground over a distance that spans a ground-temperature change $\Delta T_{\mathrm{g}}$. As the $\delta^{18} \mathrm{O}$ data comes from snow that fell over a season or more, $\Delta \sigma$ should be much less than liquid-water saturation (the maximum variation possible), and moreover should be smaller for larger $\Delta T_{\mathrm{g}}$. Reasonable values are $\Delta T_{\mathrm{g}}=10^{\circ} \mathrm{C}$ and $\Delta \sigma=0.1$. The last two factors in Eq. (17) depend on the $\alpha\left(\sigma_{\infty}\right)$ curve: for $x=0.95$, the product is 0.0016 ; for $x=1.05$, the product is larger, at 0.0092 ; and for the KF curve, the value lies between the two surface-kinetic cases, at 0.0035 . The resulting product gives variations in $\delta^{18} \mathrm{O}$ that range from 1.1 to $6.2 \%$, which are less than the observed variation $\left(\sim 7 \%\right.$ ). These observed variations in $\delta^{18} \mathrm{O}$ likely arise from a range of sources, not only supersaturation variations, so the analysis shows that surface-kinetic fractionation predicts scatter in ground-level $\delta^{18} \mathrm{O}$ that is consistent with observations.

Finally, the two surface-kinetic examples here are based on the assumption that $z$ is large, which is unlikely to hold 
over wide temperature-supersaturation regimes. Thus, unless the value of $x$ is either larger than 1.05 or less than 0.95 , the effects of surface-kinetic fractionation will deviate from the KF standard less than those analyzed here. So, within the current uncertainties of ice-crystal growth and the value of $x$, the predicted trends from surface-kinetic fractionation are consistent with observations of Antarctic snow composition.

\subsection{The need for new measurements}

As the previous section showed, having a surface-kinetic $\alpha$ value that differs from the presently used $\alpha_{\mathrm{KF}}$ means that the inferred cloud supersaturations $\sigma_{\infty}(T)$ (e.g., curve "KF" in Fig. 4) must be recalculated, but the empirical relation (Eq. 15) remains unchanged. Thus, if the empirical relation is used for a simple temperature reconstruction, then surfacekinetic fractionation changes nothing. However, a more reliable approach to past climate reconstruction involves numerous modeling considerations (see e.g., Sturm et al., 2010), including estimating how the season, the climate, and the atmospheric circulation pattern would change the $\sigma_{\infty}(T)$ relation. As the surface-kinetic theory requires a change to the inferred supersaturations that may reach $20 \%$ or more (Fig. 4), such changes to the modeled $\sigma_{\infty}(T)$ relation would affect climate reconstructions with surface-kinetic fractionation. Thus, implications of the new $\alpha$ values on past climate reconstructions are potentially significant, but presently hard to judge.

But existing measurements of $\alpha$ are inconclusive. Previous vapor-to-ice fractionation experiments are limited to measurements of $\alpha$ on largely non-facetted crystals. Specifically, Jouzel and Merlivat (1984) exposed a $-20^{\circ} \mathrm{C}$ surface to water vapor at $20^{\circ} \mathrm{C}$, conditions that produce highly dendritic frost crystal forms. Later, Uemura et al. (2005) analyzed similarly dendritic frost forms. Thus, although those experiments were appropriate for testing the KF model, they cannot be used to understand surface-kinetic fractionation on largely facetted crystals. Instead, to test this model, we need new experiments on completely facetted crystals.

Moreover, because $\alpha$ depends on both $T$ and $\sigma_{\infty}$, if we measure the dependence for both $\mathrm{HDO}$ and $\mathrm{H}_{2}^{18} \mathrm{O}$, one could then, in principle, use observed $\delta^{18} \mathrm{O}$ and $\delta \mathrm{D}$ values to infer both the deposition temperature and supersaturation of an ice sample.

\section{Conclusions}

Unlike the kinetic-fractionation (KF) theory, the surfacekinetic theory includes potentially important surface processes on facetted ice crystals. When the surface impedance to growth is low, both the kinetic and surface-kinetic models give similar predictions, showing significant deviations to equilibrium fractionation at moderate-to-high supersaturations. In contrast, when the surface impedance is compa- rable to the vapor impedance, the fractionation coefficient depends sensitively on the ratio of the deposition coefficient functions for the ordinary and isotopologue water molecules, giving results that deviate sharply from kinetic fractionation results. Such conditions should hold during the growth of facetted crystals, and since facetted crystals are common in the atmosphere, the new theory should apply to some cases in which the KF theory has previously been used. However, before the new theory can be applied to the atmosphere, we need to either measure the effect directly or experimentally determine the relevant deposition coefficient functions over a range of temperatures and supersaturations.

\section{Appendix A}

\section{The vapor impedances}

The vapor impedance depends on the crystal shape. For a spherical crystal

$Z_{\mathrm{V}} \equiv \frac{r v}{4 D}$,

where $r$ is the radius of the crystal. For a cylindrical crystal

$Z_{\mathrm{VB}}=r_{\mathrm{B}} h_{\mathrm{BE}}+r_{\mathrm{P}} h_{\mathrm{PE}} \frac{\beta_{\mathrm{P}}}{\beta_{\mathrm{B}}}$

and

$Z_{\mathrm{VP}}=r_{\mathrm{B}} h_{\mathrm{BE}} \frac{\beta_{\mathrm{B}}}{\beta_{\mathrm{P}}}+r_{\mathrm{P}} h_{\mathrm{PE}}$,

where $r_{\mathrm{B}}, r_{\mathrm{P}}, h_{\mathrm{BE}}, h_{\mathrm{PE}}$ are from Nelson, 2001, with the following slight changes. Here $r_{\mathrm{B}}=Z_{\mathrm{V}}(2 / 3 \Gamma)^{1 / 3} / 2^{1 / 2}$ and $r_{\mathrm{P}}=$ $Z_{\mathrm{V}}(2 / 3 \Gamma)^{1 / 3} \Gamma^{1 / 2}$ are normalized sizes of the top-bottom (basal) and side (prism) faces, with $\Gamma$ the column length divided by its diameter. Physically, $r_{\mathrm{B}}$ is the radius of a sphere having the same area as the basal faces of the cylinder, scaled by the distance $4 D / v$. For easier comparison to the spherical case, it is written in terms of $Z_{\mathrm{V}}$ for a sphere of the same volume. Similarly, $r_{\mathrm{P}}$ is the scaled radius of the sphere with the same area as the prism faces of the cylinder. The two $h$ functions fit

$h_{\mathrm{BE}}(\Gamma)$

$=\sqrt{2} \times 10^{-0.1315 \operatorname{Tanh}\left[0.8060\{\log (\Gamma)+0.1854\}-0.0639 \log ^{2}(\Gamma)\right]-0.3314}$

and

$h_{\mathrm{PE}}(\Gamma)$

$=0.6902 \cdot \Gamma^{-0.5+1 /\left[1.932+0.4976 \log (\Gamma)+0.1058 \log ^{2}(\Gamma)\right]}$.

(In Nelson, 2001, the $h$ values are half the above, but the product $r h$ is unchanged). Wood et al. (2001) showed that the above basis functions $h$ are very nearly the same as the corresponding basis functions for a hexagonal column of the same aspect ratio. 


\section{Appendix B}

\section{Estimated range of $x$}

Due to the complexity and unknown nature of the ice surface, one can make only crude estimates of the possible range of the deposition-coefficient ratio $x$. Now, each deposition coefficient $\beta$ is a product of two factors: the probability that a water molecule (or water isotopologue) reaches a growth step after landing on the surface and the probability of incorporating into the crystal after reaching the step. Thus, as $x$ is a ratio of the $\beta \mathrm{s}$, we can consider $x$ as the product of two ratio factors: one, the ratio of probabilities of reaching a growth step and two, the ratio of incorporation probabilities.

Assuming, as is expected, that the deposition coefficients are much less than unity, we can equate the first factor to the ratio of surface migration distances (see e.g., Yokoyama and Kuroda, 1990). The surface migration distance equals the square root of the surface diffusion constant times the mean surface residence time (Burton et al., 1951). This diffusion constant should be inversely related to the molecular mass, as it is for gas-phase diffusion (e.g., Cappa et al., 2003). Indeed, Livingston et al. (1997) found that the square root of the ratio of bulk diffusion constants of $\mathrm{HDO}$ and $\mathrm{H}_{2}^{18} \mathrm{O}$ into ordinary ice at $163 \mathrm{~K}$ equaled 1.3. Thus, if this surface-diffusion ratio dominated $x$, then we would expect $x>1$. However, surface diffusion differs from bulk diffusion and the mean residence time could instead make $x<1$ if the heavier molecule had the greater residence time.

In addition, this first factor could be highly temperature dependent. For example, measurements of the migration distance of ordinary water on the basal face of ice (Mason et al., 1963) indicated that it varied rapidly with temperature, decreasing by a factor of five when temperature decreased from -2 to $-6{ }^{\circ} \mathrm{C}$, and then increasing again by the same factor from -6 to $-12{ }^{\circ} \mathrm{C}$. If the corresponding curve for the isotopologue on regular ice is similar in shape, but shifted to higher temperature in accordance to the higher melting temperature of isotopic ice, then this factor could be as small as 0.2 or as large as 5.0. Such large deviations from unity, however, may be unlikely, and may apply only to the basal face - we have yet no corresponding measurements for the prism face of ice.

In contrast, given that the isotopologue differs from regular water, and that foreign molecules do not readily incorporate into bulk ice, we expect the second factor to produce $x>1$, though the effect may be very small. Thus, the first factor probably dominates the behavior of $x$, and it may be either less than or greater than unity, but probably not by much. Here, we consider only the possible range $0.8 \leq x \leq 1.2$.

\section{Appendix C}

\section{Analytic fit for surface impedance}

According to Eqs. (3) and (10), $Z_{\mathrm{S}}$ depends directly on $\sigma_{\mathrm{S}}$, a quantity we can know only indirectly. To estimate $\sigma_{S}$, and thus $Z_{\mathrm{S}}$, we can apply root-finding methods to Eq. (2). The result will depend on the other variables in the equation, namely the directly measurable quantities $\sigma_{\infty}, T$, and $r$, as well as surface parameters $n$ and $\sigma_{1}$. By using such a method, I found an approximate formula for $Z_{S} / Z_{V}$ in terms of these other variables. Specifically, if we use the derived parameter

$\Phi \equiv\left(\frac{\sigma_{\infty}}{\sigma_{1}}\right) Z_{V}^{\frac{1}{n}}$,

then the resulting fitted function is

$$
\frac{Z_{\mathrm{S}}}{Z_{V}} \equiv z(\Phi, n)=1.5 n \frac{\Phi^{-n /(n+1)+1 / 4}}{\log \left(1+1.5 n \Phi^{n^{2} /(n+1)+1 / 4}\right)} .
$$

Equations $(\mathrm{C} 1)$ and $(\mathrm{C} 2)$ estimate $1+z$ within $5 \%$ of the exact value for the range of atmospherically possible values of $n$ and $\Phi$, (i.e., $1 \leq n \leq 50,10^{-1} \leq \Phi \leq 10^{5}$ ), but it applies only to crystal shapes approximated by a sphere (i.e., equiaxial, facetted crystals). The equations show that when $\sigma_{1}>\sigma_{\infty}$ and $n$ is large, $z \rightarrow\left(\sigma_{\infty} / \sigma_{1}\right)^{-n} Z_{\mathrm{V}}^{-1}$, which becomes large. In contrast, $z$ decreases as $\sigma_{\infty}$ increases. Figure 1 shows both of these trends. Moreover, as $Z_{\mathrm{V}}$ increases during growth, $z$ will decrease. Finally, using Eq. (10), one can show that the reduction in supersaturation $\sigma_{\mathrm{S}} / \sigma_{\infty}$ depends only on $n$ and $\Phi$.

Acknowledgements. I thank Marcia Baker and Brian Swanson for their critical reading of the manuscript.

Edited by: T. Röckmann

\section{References}

Burton, W. K., Cabrera, N., and Frank, F. C.: The growth of crystals and the equilibrium structure of their surfaces, Philos. T. Roy. Soc. (London), A243, 299-358, 1951.

Cappa, C. D., Hendricks, M. B., DePaolo, D. J., and Cohen, R. C.: Isotopic fractionation of water during evaporation, J. Geophys. Res. 108, 4525-4534, 2003.

Dansgaard, W., Johnsen, S. J., Moeller, J., and Langway Jr., C. C.: One thousand centuries of climatic record from Camp Century on the Greenland ice sheet, Science, 166, 377-380, 1969.

DePaolo, D. J.: Surface kinetic model for isotopic and trace element fractionation during precipitation of calcite from aqueous solution, Geochim. Cosmochim. Acta 75, 1039-1056, 2011.

Fisher, D. A.: Remarks on the deuterium excess in precipitation in cold regions, Tellus 43B, 401-407, 1991.

Frank, F. C.: Snow Crystals, Contemp. Phys., 23, 3-22, 1982.

Fujita, K. and Abe, O.: Stable isotopes in daily precipitation at Dome Fuji, East Antarctica, Geophs. Res. Lett., 33, L18503, doi:10.1029/2006GL026936, 2006. 
Johnsen, S. J., Dansgaard, W., and White, J. W. C.: The origin of Arctic precipitation under present and glacial conditions, Tellus 41B, 452-468, 1989.

Jouzel, J.: Isotopes in Cloud Physics: Multiphase and Multistage Condensation Processes, Handbook of Environmental Isotope Geochemistry, Vol. 2. The Terrestrial Environment, edited by: Fritz, B. P and Foutes, J. Ch., Elsevier, New York, 61-112, 1986.

Jouzel, J. and Merlivat, L.: Deuterium and Oxygen 18 in Precipitation: Modeling of the Isotopic Effects During Snow Formation, J. Geophys. Res., 89, 11749-11757, 1984.

Kuroda, T.: Rate Determining Processes of Growth of Ice Crystals from the Vapour Phase. Part I: Theoretical Consideration, J. Meteorol. Soc. Japan, 62, 552-561, 1984.

Lawson, R. P., Baker, B. A., Zmarzly, P., O’Connor, D., Mo, Q., Gayet, J.-F., and Shcherbakov, V.: Microphysical and Optical Properties of Atmospheric Ice Crystals at South Pole Station, J. Appl. Meteor. Clim. 45, 1505-1524, 2006.

Langway Jr., C. C.: The history of early polar ice cores, ERCD/CRREL TR-08-1, US Army Corps of Engineers, 2008.

Livingston, F. E., Whipple, G. C., and George, S. M.: Diffusion of HDO into Single-Crystal $\mathrm{H}_{2}^{16} \mathrm{O}$ Ice Multilayers: Comparison with $\mathrm{H}_{2}^{18} \mathrm{O}$, J. Phys. Chem. B, 101, 6127-6131, 1997.

Majoube, M.: Fractionation factor of ${ }^{18} \mathrm{O}$ between water vapour and ice, Nature, 226, p. 1242, 1970.

Mason, B. J., Bryant, G. W., and van den Huevel, A. P.: The growth habits and surface structure of ice crystals, Phil. Mag., 8, 505526, 1963.

Masson-Delmotte, V., Hou, S., Ekaykin, A., et al.: A Review of Antarctic Surface Snow Isotopic Composition: Observations, Atmospheric Circulation, and Isotopic Modeling, J. Climate, 21, 3359-3387, 2008.

Merlivat, L.: Molecular diffusivities of $\mathrm{H}_{2}^{16} \mathrm{O}, \mathrm{HD}^{16} \mathrm{O}$ and $\mathrm{H}_{2}^{18} \mathrm{O}$ in gases, J. Chem. Phys. 69, 2864-2871, 1978.
Merlivat, L. and Nief, G.: Fractionnement isotopique lors des changement d'etat solide-vapeur et liquide-vapeur de l'eau a des temperatures inferieures a $0{ }^{\circ} \mathrm{C}$, Tellus 1., XIX, 122-127, 1967.

Nelson, J.: Growth mechanisms to explain the primary and secondary habits of snow crystals, Phil. Mag. A, 81, 2337-2373, 2001.

Nelson, J.: Branch Growth and Sidebranching in Snow Crystals, Crystal Growth \& Design 5, 1509-1525, 2005.

Nelson, J. and Baker, M. B.: A New Theoretical Framework for Studies of Vapor Growth and Sublimation of Small Ice Crystals in the Atmosphere, J. Geophys. Res. 101, 7033-7047, 1996.

Nelson, J. and Knight, C.: Snow Crystal Habit Changes Explained by Layer Nucleation, J. Atmos. Sci., 55, 1452-1465, 1998.

Salamatin, A. N., Ekaykin, A. A., and Lipenkov, V. Ya.: Modelling isotopic composition in precipitation in Central Antarctica, Mater. Glyatsiologicheskih Issled, 97, 24-34, 2004.

Sturm, C., Zhang, Q., and Noone, D.: An introduction to stable water isotopes in climate models: benefits of forward proxy modelling for paleoclimatology, Clim. Past, 6, 115-129, doi:10.5194/cp-6-115-2010, 2010.

Takahashi, T. Endoh, T., Wakahama, G., and Fukuta, N.: Vapor diffusional growth of free-falling snow crystals between -3 and $-23{ }^{\circ} \mathrm{C}$, J. Meteorol. Soc. Jpn., 69, 15-30, 1991.

Uemura, R., Matsui, Y., Yoshida, N., Abe, O., and Mochizuki, S.: Isotopic fractionation of water during snow formation: Experimental evidence of kinetic effect, Polar Meteorol. Glaciol., 19, 1-14, 2005.

Wood, S. E., Baker, M. B., and Calhoun, D.: New model for the vapor growth of hexagonal ice crystals in the atmosphere, J. Geophys. Res., 106, 4845-4870, 2001.

Yokoyama, E. and Kuroda, T.: Pattern formation in growth of snow crystals occurring in the surface kinetic process and the diffusion process, Phys. Rev. A, 41, 2038-2049, 1990. 